\title{
Nonlinear Spectroscopy of Cold, Trapped Atoms
}

\author{
J. Zachorowski*, T. Brzozowski, T. PaŁasz, M. Zawada \\ AND W. GAWLIK \\ Instytut Fizyki M. Smoluchowskiego, Uniwersytet Jagielloński \\ Reymonta 4, 30-059 Kraków, Poland
}

\begin{abstract}
We report on our studies of atoms contained in a magneto-optical trap using the nonlinear spectroscopy methods. Absorption and four-wave mixing signals are recorded for the probe frequency near the cooling transition frequency and the two methods are compared. The differences in the signal structure and their sensitivity on external conditions are discussed. It is revealed that central feature of these spectra consists of several contributions of different origin.
\end{abstract}

PACS numbers: $32.80 . \mathrm{Pj}, 42.65 . \mathrm{An}, 42.65 . \mathrm{Hw}$

\section{Introduction}

Cold samples of trapped atoms form very attractive objects for spectroscopic research. Their main characteristic is the absence of an inhomogeneous broadening. The popular way to study the properties of cold, well-isolated atoms is such that the atoms are gathered in a magneto-optical trap (MOT) [1] and cooled there, but during the actual measurement when they are subject to pump and probe laser beams, the trapping light, and often also the quadrupole magnetic field, are switched off. While such experiments play a very important role for better understanding the quantum processes of light interaction with multilevel atoms, they provide little information on the atom dynamics in a working trap. At the same time, spectroscopy provides also a means of studying properties and dynamics of cold, trapped atoms in situ. In this aspect the spectroscopic measurement can be used to study the behavior of atoms in the trap and effective parameters of the trap itself. Therefore, such experiments should be performed while the trap is active and all the fields present.

*corresponding author; e-mail: zachorowski@if.uj.edu.pl 
First experiments on nonlinear absorption of trapped atoms have been performed by Tabosa et al. [2] with rubidium and by Grison et al. [3] with cesium atoms. Studies of absorption spectra of atoms gathered in the trap, but after switching the trap beams off, were performed by Mitsunaga et al. [4], Lipsich et al. [5], and Chen et al. [6]. Four-wave mixing (FWM) spectroscopy of atoms in a MOT has started with the works of Lounis et al. [7, 8], and Hemmerich et al. [9] and continued by Tabosa et al. [10-12] and Lezama et al. [13].

Our aim is to establish methods of a real-time diagnostics of atoms in a working MOT. We have thus undertaken studies of the nonlinear spectra with the use of both absorption and FWM spectroscopy. The measurements were performed in the configuration where the trapping beams are present and play the role of pump. The quadrupole magnetic field were neither switched off, i.e., the recorded spectra characterize a working MOT rather than an unperturbed cloud of cold atoms.

\section{Magneto-optical trap}

Let us recall briefly the working principles of the magneto-optical trap (Fig. 1). Atoms in a light field are subject to two kinds of optical forces: the spontaneous or light pressure force due to the gradient of the light field phase $\boldsymbol{F}_{\mathrm{S}} \propto \nabla \Phi(r)$ and the dipole force due to the gradient of the field amplitude $\boldsymbol{F}_{\mathrm{D}} \propto \nabla E(r)$. The two kinds of forces differ in that one is dissipative and the other reactive, they have different dependence on the light frequency around the atomic resonance line. A combination of six light beams of frequency $\nu_{\mathrm{L}}$, counter propagating along the three

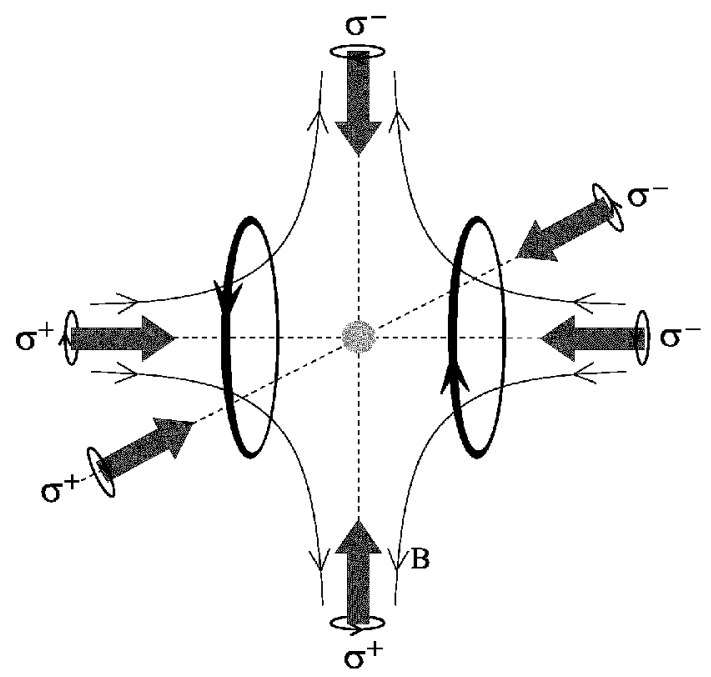

Fig. 1. Scheme of the magneto-optical trap. Six light beams of circular polarization $\sigma^{+} / \sigma^{-}$exert light-pressure force on atoms in the magnetic quadrupole field $\boldsymbol{B}$ produced by two coils of opposite current flow (thick loops). 
orthogonal spatial directions, and detuned by about 1-3 natural widths $\Gamma$ below the atomic transition (red detuning) give a resulting force which has a viscous character, $\boldsymbol{F}(v) \propto-v$. This force cools atoms and forms the so-called optical molasses. When quadrupole magnetic field is produced where the beams are crossing and proper circular polarization of the beams is used, the force becomes position dependent, $\boldsymbol{F}(\boldsymbol{r}) \propto-\boldsymbol{r}$, and optical force provides the restoring force needed to form a trap.

The cloud of cold atoms stored in the MOT contains $10^{6}-10^{8}$ atoms and has the size of a fraction of a mm. The maximum density is limited to $10^{10}-10^{12}$ atoms $/ \mathrm{cm}^{3}$. The kinetic temperature of the atomic sample is of the order of the Doppler limit temperature which is $0.1-1 \mathrm{mK}$. This temperature corresponds to the atomic velocities of the order of $10 \mathrm{~cm} / \mathrm{s}$. Even lower temperatures can be obtained by sub-Doppler cooling after switching the magnetic field off.

The most important feature of the atoms in the trap from the spectroscopy's point of view is that atomic Doppler width is smaller than natural width of the transition used for cooling the atoms, $\Delta_{\mathrm{D}} \ll \Gamma$. This constitutes a sample of unusual absorption properties where many interesting effects, normally blurred by the Doppler effect, show up easily. This sample possesses also some very interesting characteristics as a medium for the wave-mixing experiments. Since the Doppler width is smaller than the natural width, all atoms contribute to the signal, moreover, for the almost immobile atoms the "grating washing" effect [14] is strongly reduced, which allows for relatively big angles between the pump and the probe beams. On the other hand, the number of atoms and the size of the sample is small, so the achieved four-wave mixing efficiency reaches $1 \%$. The slow decrease in the signal due to the movement of the atoms has been used by Mitsunaga et al. [15] to measure the temperature of the atoms via the time-resolved four-wave mixing.

Our experiment was performed with ${ }^{85} \mathrm{Rb}$ atoms trapped in a vapor-loaded MOT. For the details of the trap see [16]. The axial gradient of the quadrupole magnetic field was $6 \mathrm{Gs} / \mathrm{cm}$. The laser beams used for trapping had waist of $4 \mathrm{~mm}$ and carried the typical power $I_{\mathrm{L}}=14 \mathrm{~mW} / \mathrm{cm}^{2}$ each. This intensity was varied in the experiment, which resulted in the changes of the number of stored atoms, the cloud density, and temperature. The trapping laser was locked to the atomic resonance and tuned below the frequency $\nu_{3-4}$ of the cooling transition $F_{\mathrm{g}}=3 \rightarrow F_{\mathrm{e}}=4$ of the ${ }^{85} \mathrm{Rb} \mathrm{D}_{2}$ line with the use of an acousto-optical modulator (AOM). The actual detuning $\delta=\nu_{\mathrm{L}}-\nu_{3-4}$ was varied between -6 and $-30 \mathrm{MHz}(-1$ to $-5 \Gamma)$ in the experiment. To avoid the escape of atoms into the other hyperfine ground level a weak repumping beam, resonant with the $F_{\mathrm{g}}=2 \rightarrow F_{\mathrm{e}}=3$ transition, was superimposed on the trap. The measured temperature of atoms in the trap was $0.75 \mathrm{mK}$ for laser detuning of $\delta=-2 \Gamma$ and for low trapping intensity of $2.9 \mathrm{~mW} / \mathrm{cm}^{2}$ in each beam. This corresponds well to the theoretical predictions of the temperature for these parameters. 


\section{Wide scan}

Our studies were performed in a setup schematized in Fig. 2. The six trap beams acted as the pump field. A weak beam of linear polarization and of frequency $\nu_{\mathrm{p}}$ was used as a probe of the trapped atom absorption or for inducing the FWM signal. The probe beam made an angle of about $20^{\circ}$ to one of the trapping beams and was focused to a waist of $100 \mu \mathrm{m}$. Detector 1 (see Fig. 2) recorded the absorption spectrum and a beam splitter inserted in the probe beam directed the generated phase-conjugated beam (FWM beam) to detector 2. The lock-in detection scheme was used to improve the signal-to-noise ratio in the wave-mixing measurements.

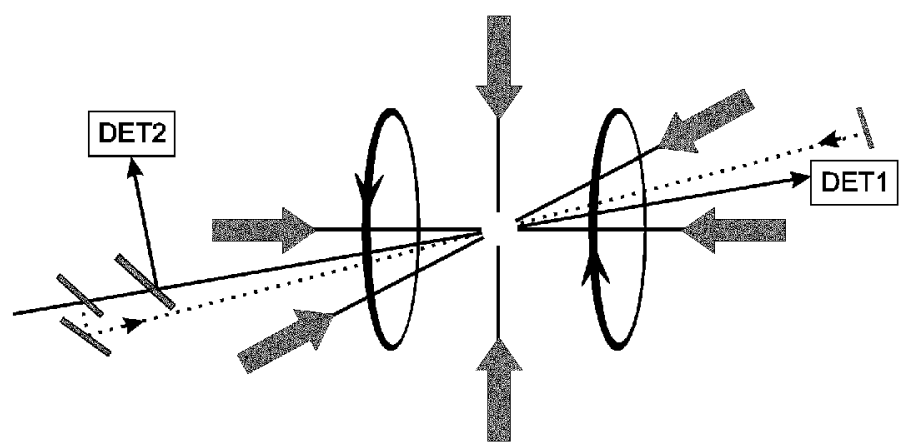

Fig. 2. Experimental setup illustrating the probe beam and two detectors. DET1 records the absorption signal and DET2 measures the intensity of the FWM beam reflected from the beam splitter. The dotted line indicates optional pump beams used in degenerate FWM measurements.

In the recordings of the wide scans of absorption and FWM, covering all the lines of transition from the $F_{\mathrm{g}}=3$ ground state, we derived the probe beam from a separate diode laser, tuned independently. As the relative frequency difference between the pump laser and the probe laser was not controlled there is an additional broadening of about $5 \mathrm{MHz}$ in the spectra.

\subsection{Absorption}

A typical plot of an absorption spectrum is presented in Fig. 3. It shows absorption changes around all allowed hfs components: $F_{\mathrm{g}}=3 \rightarrow F_{\mathrm{e}}=2,3$, and 4 . Those associated with the $3 \rightarrow 2$ and $3 \rightarrow 3$ transitions have a form of asymmetric doublets with one strong component slightly above the unperturbed resonance frequency (blue-shifted) and one much weaker, red-shifted component. In Fig. 3, the red-shifted component of the $F_{\mathrm{g}}=3 \rightarrow F_{\mathrm{e}}=2$ doublet is too weak to be resolved and can be seen only as a broadening of the low-frequency wing of the absorption line. Around the $3 \rightarrow 4$ hfs transition, the absorption signal has a 


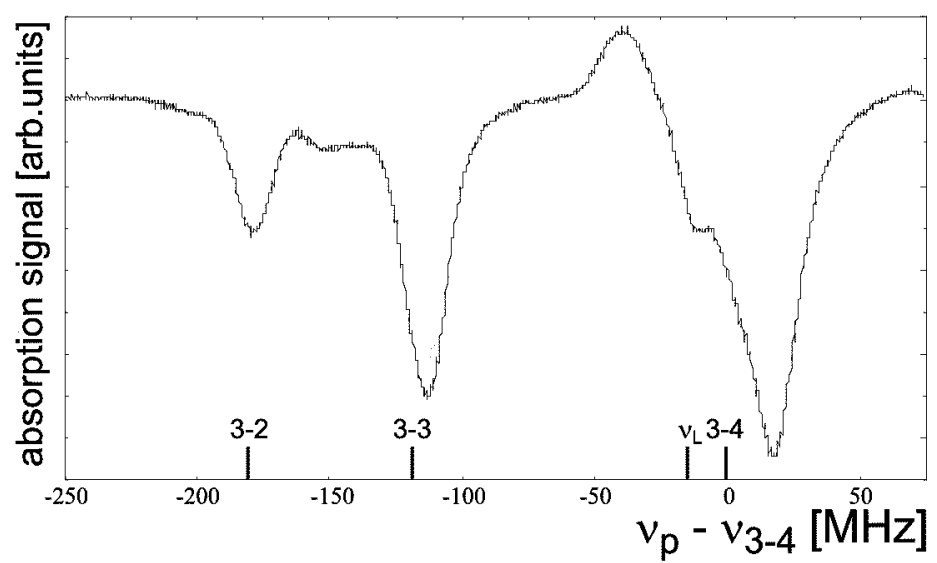

Fig. 3. Absorption, wide scan over all hfs components. The trapping laser is at $\nu_{\mathrm{L}}=$ $\nu_{3-4}-12 \mathrm{MHz}$. Intensity of each trapping beam is $14.4 \mathrm{~mW} / \mathrm{cm}^{2}$.

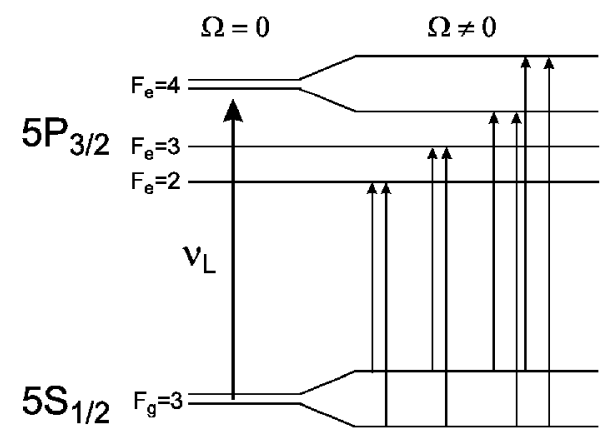

Fig. 4. Energy levels of Rb atoms dressed by the strong cooling beam for $\Omega=0$ and $\Omega \neq 0$. The resonant transitions in the dressed atom are indicated. A weak perturbation of levels $F_{\mathrm{e}}=2$ and 3 is neglected in the figure.

distinct triple structure consisting of two side bands: one showing the probe gain at the low-frequency side of $\nu_{\mathrm{L}}$ and another, absorptive one on the blue side of $\nu_{3-4}$ plus a central feature at the pump laser frequency $\nu_{\mathrm{L}}$. All these resonances can be easily explained in terms of dressed atom states (Fig. 4).

\subsection{FWM spectra}

FWM spectra differ strongly from the absorption signals. While absorption exhibits visible resonances corresponding to all allowed hfs transitions irrespectively of the pump beam frequency and power (in particular, even without any pump beam an unperturbed Doppler-free linear absorption can be recorded), this is not the case with the nonlinear FWM signals. We have observed that with the trap beams kept at about $1-3 \Gamma$ below $\nu_{3-4}$ the wave mixing occurs only when the probe is tuned close to this transition. The absence of FWM at other hfs components is due to very big detuning of the pump beams from the resonance with 
the $F_{\mathrm{g}}=3 \rightarrow F_{\mathrm{e}}=2,3$ transitions. FWM is a highly nonlinear process strongly enhanced by tuning all involved light beams to resonance with appropriate intermediate atomic states. When the pump frequency is close to $\nu_{3-4}$, it is too far from other hfs states to give a noticeable contribution to the FWM at other transitions.

In order to see the FWM signal at each allowed hfs component, we used the technique of degenerate FWM (DFWM). The second laser provided not only a weak probe beam but also two stronger, counterpropagating beams acting as additional pumps. They were directed onto the trapped cloud under $1.6^{\circ}$ angle to the probe beam and about $20^{\circ}$ to one of the trapping beams. In this way, the phase-conjugated signals due to DFWM could be recorded for atoms simultaneously illuminated by trapping beams. Figure 5 shows a typical spectrum obtained

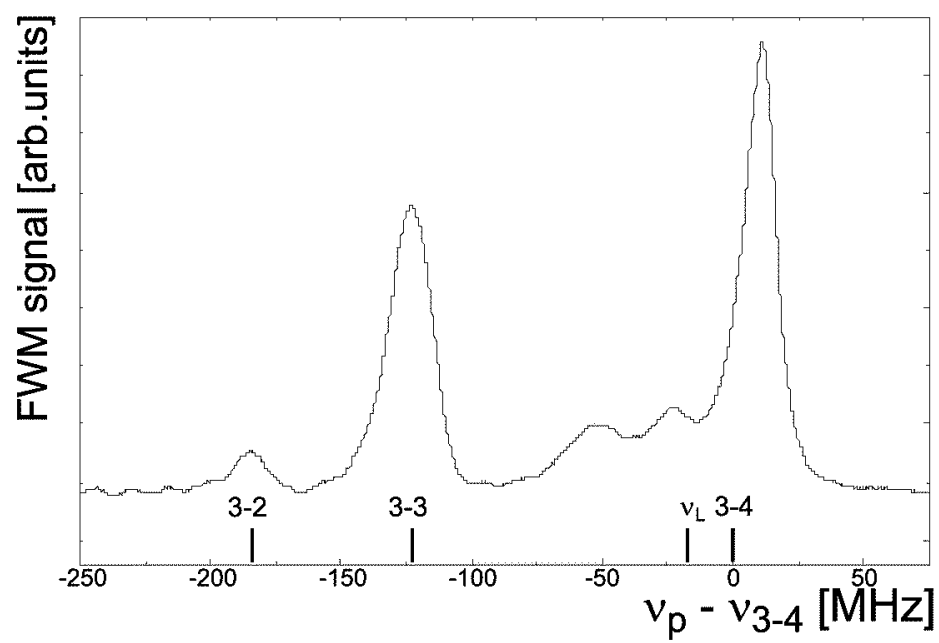

Fig. 5. Degenerate four-wave mixing signal, wide scan over all hfs components. The trapping laser is at $\nu_{\mathrm{L}}=\nu_{3-4}-15.7 \mathrm{MHz}$. Intensity of each trapping beam is $14.4 \mathrm{~mW} / \mathrm{cm}^{2}$ and of each additional pump beam is $0.7 \mathrm{~mW} / \mathrm{cm}^{2}$.

while scanning the second laser (the probe and two additional pump beams) over the $F_{\mathrm{g}}=3 \rightarrow F_{\mathrm{e}}=2,3$, and 4 transitions. DFWM signal occurs around each of the hfs components, similarly to the absorption spectra of Fig. 3 . The intensity of the additional counterpropagating beams was up to $0.70 \mathrm{~mW} / \mathrm{cm}^{2}$ per each beam, i.e., much less than the $14.4 \mathrm{~mW} / \mathrm{cm}^{2}$ typical intensity of each of the trap beams. For this reason, the presence of the additional beams does not add much perturbation to this already imposed by the trap beams on the atomic structure and dynamics. We have verified this by recording the absorption spectra with these additional beams (not shown here). Almost no distortion of the spectra was seen except for about $50 \%$ reduction of the absorption depth. Most likely this reduction was due to additional saturation caused by more efficient optical pumping by the two extra beams. This extra saturation reduces also resolution of the DFWM spectra. In 
particular, weak components of the doublets in the $3 \rightarrow 2$ and $3 \rightarrow 3$ components are now seen only as the line asymmetry. Apart from that, the resolution of the wide scans is limited mainly by the relative noise between two independent lasers.

\section{Narrow scan}

To get better resolution around the trapping transition we repeated the scans but with the probe laser locked to the trapping laser (injection locking). We used two AOMs to control the frequency of the probe laser: one tunable in a double passage to shift the frequency of the injected laser beam and thus to set the probe frequency and one with fixed frequency. This resulted in perfect phase

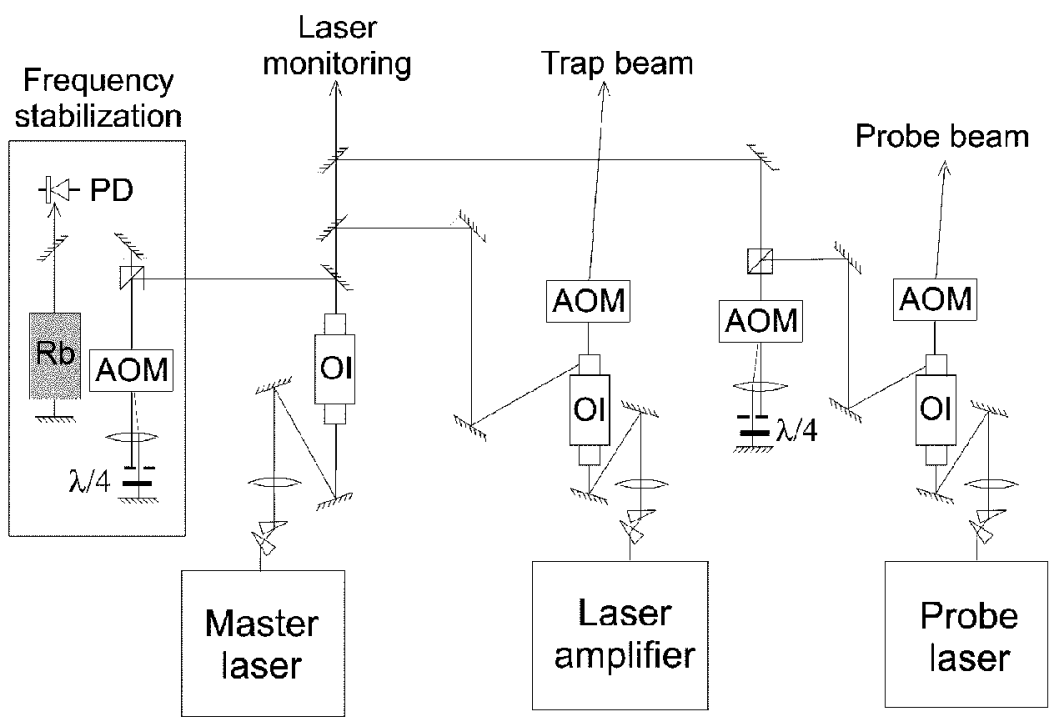

Fig. 6. Scheme of the laser system. OI - optical isolator, AOM - acousto-optical modulator, $\mathrm{PD}$ - photodiode, $\lambda / 4$ - quarter-wave plate.

correlation between the pump (trap) and probe beams and allowed recording of spectral structures well below the $6 \mathrm{MHz}$ natural width of the Rb lines and even below the laser line width. The parameters of the AOMs limited the possible scan range of about $\pm 60 \mathrm{MHz}$ around the pump frequency $\nu_{3-4}$. The scheme of the laser system is shown in Fig. 6.

\subsection{Absorption}

The high-resolution absorption spectrum of strongly driven cold atoms is presented in Fig. 7. If we analyze the spectrum in terms of a dressed two-level atom in the vicinity of the strong pump frequency we should expect a triplet structure consisting of an absorptive feature close to $\nu_{3-4}$, of a three-photon gain 
feature close to $2 \nu_{\mathrm{L}}-\nu_{3-4}$, and of an asymmetric component at $\nu_{\mathrm{L}}$. These AC Stark effect side bands should occur close to $\nu_{\mathrm{L}} \pm \Omega^{\prime}$, where $\Omega^{\prime}=\sqrt{\Omega^{2}+\delta^{2}}$ is the generalized Rabi frequency for the laser detuning $\delta$ [17]. Our spectrum resembles roughly such a spectrum of a two-level dressed atom. There are, however, important differences. First, the central feature has much bigger amplitude, smaller width, and even a different sign of asymmetry than in the absorption spectrum of two-level atoms. It has a complex sub-natural structure. The second difference concerns the absorptive and gain side bands, which appear much broader and are not symmetrically positioned around $\nu_{\mathrm{L}}$.

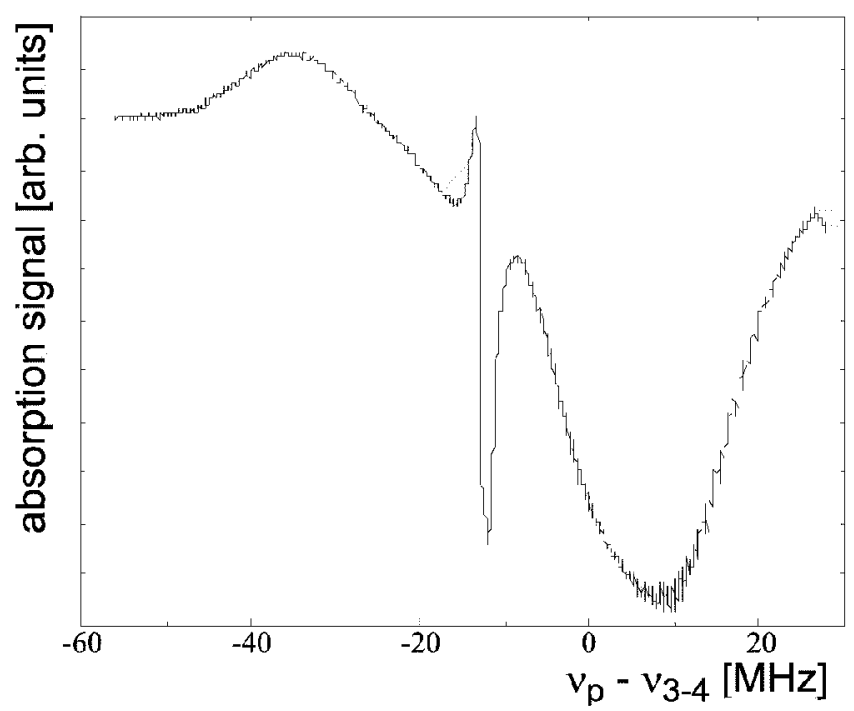

Fig. 7. Absorption, narrow scan around the trapping laser frequency. The trapping laser is at $\nu_{\mathrm{L}}=\nu_{3-4}-13 \mathrm{MHz}$. Intensity of each trapping beam is $6.4 \mathrm{~mW} / \mathrm{cm}^{2}$.

Please note that the difference in shape of the central feature at $\nu_{\mathrm{L}}$ frequency between the recordings of Fig. 3 and Fig. 7 is due to the poor resolution of the former, obtained with independent (unlocked) probe laser. We have verified that this is indeed the cause: we have numerically averaged the spectrum of Fig. 7 over the $5 \mathrm{MHz}$ relative jitter of the two lasers and obtained a spectrum very closely resembling the one in Fig. 3, with shallow and inverted central feature.

The positions of side resonances show asymmetry. We note that the low-frequency, gain side band is more displaced from $\nu_{\mathrm{L}}$ than the high-frequency, absorption side band. There are two reasons for this: one is that our atom is by no means a two-level system. It has instead several Zeeman levels and a related spread of dipole moments of individual transitions. The second reason is that there exists spatial inhomogeneity of the net trapping light field due to interference of beams intersecting in the trap, which involves periodic intensity and polarization 


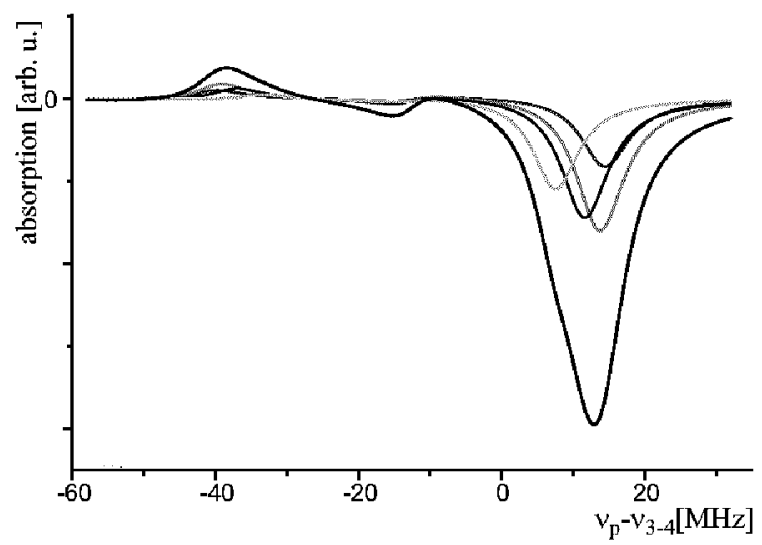

Fig. 8. Absorption spectrum due to four, Zeeman degenerate, two-level substructures with different dipole moments. The actual values of these moments correspond to the $\Delta m=0$ transitions in the rubidium $F_{\mathrm{g}}=3 \rightarrow F_{\mathrm{e}}=4$ line.

variations. Therefore, even in the simplest case when the local light polarizations of the pump and probe fields allow decomposition of the driven transition into several two-level substructures (equal circular or parallel linear polarizations), the absorption spectrum is a superposition of several spectra of two-level subsystems characterized by different dipole moments [5]. The crucial point is that the absorption and gain side bands show different amplitude dependence on the Rabi frequency. As the dipole moment of the transition increases, the gain side band also increases but the absorption one saturates. Thus, even if the side-band positions within individual triplet substructures are perfectly symmetric, the resulting superposition spectrum shows a clear asymmetry: the absorption side band is shifted towards $\nu_{\mathrm{L}}$, while the gain side band is shifted towards higher detuning. To show this effect we have modelled the absorption signal as a sum of contributions from different $\pi$-polarization transitions on the $F_{\mathrm{g}}=3 \rightarrow F_{\mathrm{e}}=4$ transition in ${ }^{85} \mathrm{Rb}$. The plot in Fig. 8 shows the same behavior as the experimental recordings.

\subsection{Four-wave mixing}

Figure 9 shows the spectrum of the near-degenerate four-wave mixing in the setup shown in Fig. 2 without the extra pump beams. The spectrum has a narrow central resonance and two side bands. These side bands have the same origin as those seen in absorption spectra, i.e., AC Stark effect, and should appear close to $\nu_{\mathrm{L}} \pm \Omega^{\prime}$. The asymmetry of side bands positions is opposite to the absorption case: the low-frequency side band is closer to the pump-laser frequency than the high-frequency one. This asymmetry cannot be caused by the different intensity dependence of the two side bands, as for the two-level atom the amplitudes of both FWM side bands scale in the same way [18] and thus the signal, which is a sum of two-level substructures, remains symmetrical. This holds also for more complicated 


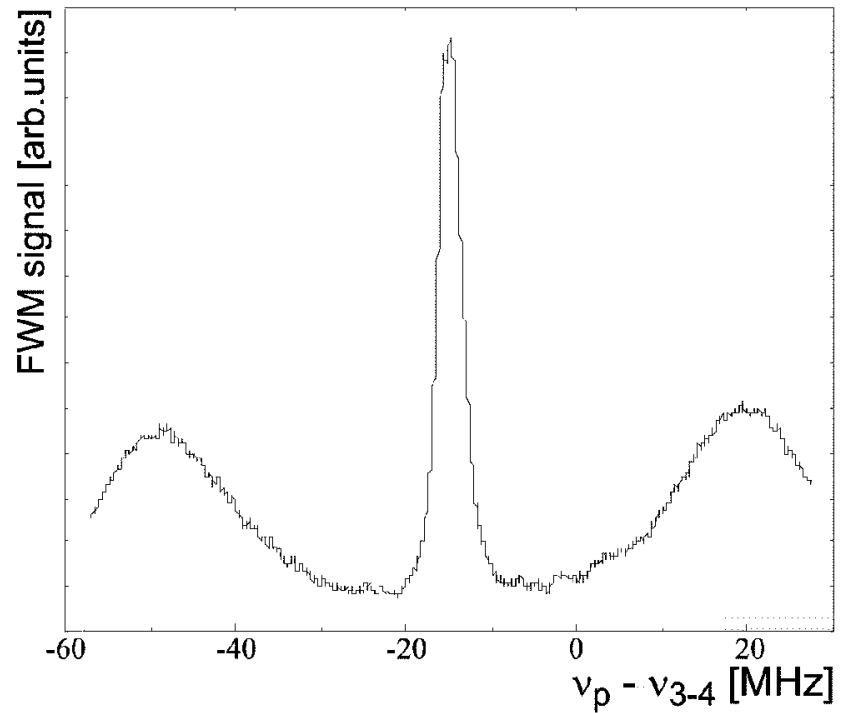

Fig. 9. Near-degenerate four-wave mixing, narrow scan around the trapping laser frequency. The trapping laser is at $\nu_{\mathrm{L}}=\nu_{3-4}-16 \mathrm{MHz}$. Intensity of each trapping beam is $14.4 \mathrm{~mW} / \mathrm{cm}^{2}$.

polarization cases. We attribute the observed asymmetry to the influence of the AC Stark perturbed absorption. In consequence, the resonant absorption shifts the position of the blue side band maximum towards higher frequencies, as seen in Fig. 9.

\section{Central resonance}

The central resonance appears for the probe frequency being very close (of the order of a few $\mathrm{MHz}$ ) to the frequency of the trap (pump) laser. It differs significantly from the case of a dressed two-level model, which predicts a dispersive shape of width $\Gamma$, while the actual experiment gives a more complicated shape. The spectra of Fig. 7 show already that the resonance width is below the natural width $\Gamma$, and its asymmetry is reversed. An even narrower scan presented in Fig. 10 shows a substructure with resonances a few hundred kilohertz apart. Please note that to record such a narrow structure it was necessary to use the probe laser injection locked to the pump laser and tuned with acousto-optical modulators.

Let us consider the situation of the $\pi$-polarized pump beam and the $\sigma$-polarized probe. Distances between the resonances correspond to the spacing between the light-shifted magnetic sublevels of the ground state. The resonances result from the Raman transitions involving pump and probe photons as suggested in [3] (Fig. 11). Due to the differences in population of the sublevels, the probe beam is 


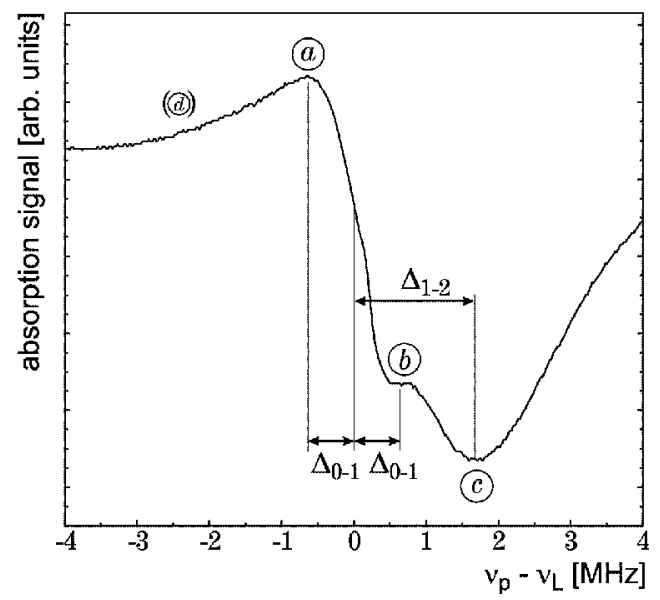

Fig. 10. Absorption signal showing only the central resonance of Fig. 7. The trapping laser is at $\nu_{\mathrm{L}}=\nu_{3-4}-14 \mathrm{MHz}$. Intensity of each trapping beam is $14 \mathrm{~mW} / \mathrm{cm}^{2}$.

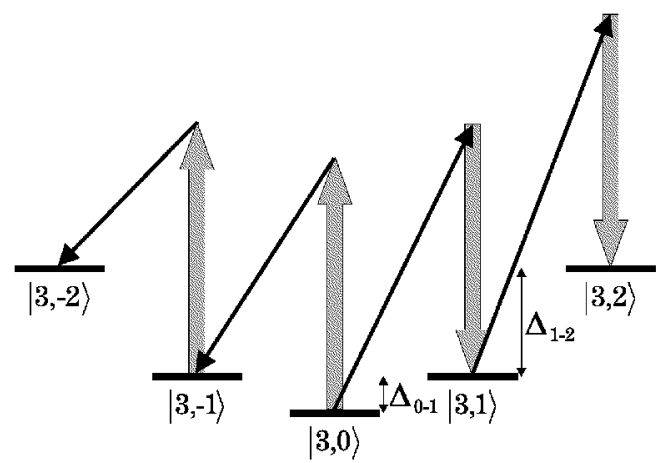

Fig. 11. Scheme of the Raman transitions for the Zeeman sublevels of the $F_{\mathrm{g}}=3$ ground level. Degeneracy of the sublevels is lifted by the light-shift effect due to the strong pump (trapping) field. Thick arrows represent $\Delta m=0$ transitions caused by the pump field, thin arrows represent $\Delta m=+1$ transitions induced by the probe field.

amplified (for positive detuning) or absorbed (for negative detuning). The positions of the Raman resonances depend on the pump light intensity and on the relevant Clebsch-Gordan (C-G) coefficients. The resonances of Fig. 10 have been associated with the transitions between states $|3,0\rangle \leftrightarrow|3,1\rangle$ and $|3,0\rangle \leftrightarrow|3,-1\rangle-$ resonances $a$ and $b$, respectively, and transitions $|3,1\rangle \leftrightarrow|3,2\rangle$ and $|3,-1\rangle \leftrightarrow|3,-2\rangle-$ resonance $c$ and its barely visible counterpart $d$. With the increase in the pump intensity the light shift increases as $\Omega^{2} / \delta$ for low perturbation, when $\Omega^{2} \ll \Gamma^{2} / 4+\delta^{2}$, or linearly with $\Omega$ for strong fields, when $\Omega^{2} \gg \Gamma^{2} / 4+\delta^{2}$. The plot of frequency of the resonance $c$ vs. the frequency of $a$ should be a straight line of slope given by the ratio of the $\mathrm{C}-\mathrm{G}$ coefficients (low perturbation) or by the square of the 
coefficients (strong perturbation). For rubidium atoms and the studied transition the two limiting cases of low and strong perturbation give 3 and 3.22 , respectively. The plot of Fig. 12 shows our experimental points which fit the straight line with slope 3.16, corresponding well to the value calculated for the range of intensities used in the experiment. This proves that the observed resonances are indeed the transitions between light-shifted magnetic sublevels.

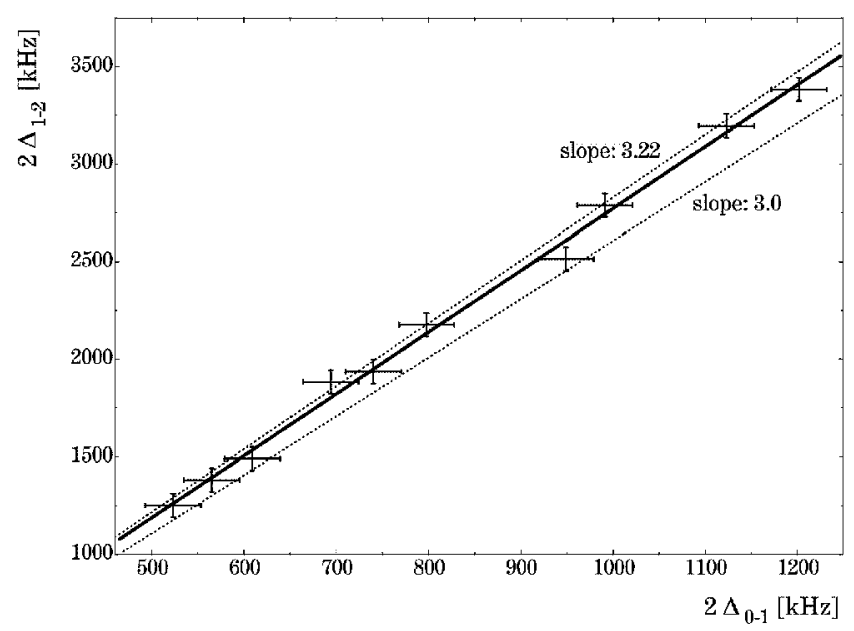

Fig. 12. Relative position of the components of the central resonance: position of the $c$ resonance vs. the one of $a$ and $b$. The two broken lines show two limiting cases: of weak field limit, when light shifts scale as $\Omega^{2} / \delta$, and of strong field limit, when light shifts become proportional to $\Omega$. The solid line is a linear fit to the experimental points with slope $3.16 \pm 0.08$. The pump beam detuning is $-14 \mathrm{MHz}$.

We have recorded also the four-wave mixing spectra around the trap laser frequency simultaneously with the absorption (Fig. 13). The FWM signal exhibits very narrow resonances at a distance of a few hundred kilohertz from the pump laser frequency that are barely visible in the absorption spectrum. One of the possible explanations of these resonances is the effect of optical lattices (see the review paper [19]). Six trapping beams create a periodic spatial modulation of intensity and/or polarization of the light field. Thus created dipole forces act on atoms and at low enough temperature of the atoms may lead to their localization in the minima of the potential and to the quantization of atomic motion. Assuming a harmonic potential, the vibrational atomic energy levels are equally spaced by a few hundred kilohertz. As the probability of tunnelling of the localized atom to the neighboring site is low, one can observe Raman transitions between the vibrational energy levels producing very narrow resonances. 


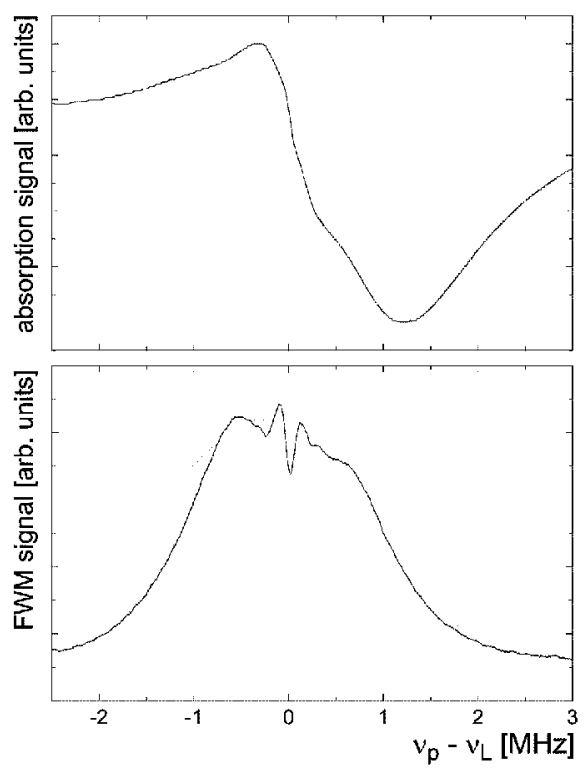

Fig. 13. Central resonance in absorption (top) and FWM spectra (bottom) recorded simultaneously.

Another effect that might show up in the signals is the recoil resonance [20]. It results from the momentum transfer between atoms and photons of the pump and probe beams. It has a quasi-dispersive shape of the Gauss curve derivative. Its width appears to be comparable to the width of the above discussed Raman resonances due to transitions between vibrational energy levels of atoms localized in optical lattices and of the same order as the experimentally observed resonances. This makes the discrimination of particular components difficult.

As noted above, the well-resolved resonances visible in the four-wave mixing spectra of Fig. 13 can only be noticed as subtle kinks in the shape of absorption signal. This proves the superior sensitivity of the wave mixing as compared to absorption in the studies of optical lattices. Nevertheless, only the simultaneous recording of absorption and FWM spectra gives a complete information on the behavior of atoms in the MOT. Some of the features of the experimentally observed spectra can be explained by the invoked theory, the others are still unexplained. In particular, it is noticeable that there is no full analogy in the positions of resonances in absorption and FWM spectra: although the two processes have different characters, the resonance condition should be the same in both cases. The full dependence of the signal shape on the probe polarization deserve further studies as well as the role of the magnetic field of the trap. 


\section{Conclusions}

In this work we have studied cold atoms in the working magneto-optical trap by the nonlinear spectroscopy methods. The absorption and four-wave mixing spectra have been recorded. The origins of the asymmetry of the AC Stark effect side bands have been discussed and attributed to the multilevel structure and spatial inhomogeneity of the field in the case of absorption and to the influence of absorption in the FWM case. The physical effects responsible for the central resonance have been presented. We have noted the differences in the sensitivity of absorption and four-wave mixing.

Nonlinear spectra of trapped atoms form a very useful diagnostics method of the trap conditions. The FWM spectroscopy appears here most suitable. It is less affected by the multilevel atomic structure and allows a more accurate determination of the strength of the light field interaction. Moreover, FWM spectra reveal very subtle effects related to optical lattices and atomic recoil.

\section{Acknowledgments}

The authors wish to thank G. Grynberg and S.G. Odoulov for valuable discussions on the FWM spectra. This work is supported by the State Committee for Scientific Research grant No. 5 P03B 01516.

\section{References}

[1] E.L. Raab, M. Prentiss, A. Cable, S. Chu, D.E. Pritchard, Phys. Rev. Lett. 59, 2631 (1987).

[2] J.R.W. Tabosa, G. Chen, Z. Hu, R.B. Lee, H.J. Kimble, Phys. Rev. Lett. 66, 3245 (1991).

[3] D. Grison, B. Lounis, C. Salomon, J.-Y. Courtois, G. Grynberg, Europhys. Lett. 15, 149 (1991).

[4] M. Mitsunaga, J. Mukai, K. Watanabe, T. Mukai, J. Opt. Soc. Am. B 13, 2696 (1996).

[5] A. Lipsich, S. Barreiro, A.M. Akulshin, A. Lezama, Phys. Rev. A 61, 053803 (2000).

[6] Y.-C. Chen, Y.-W. Chen, J.-J. Su, J.-H. Huang, I.A. You, Phys. Rev. A 63, 043808 (2001).

[7] B. Lounis, P. Verkerk, J.-Y. Courtois, C. Salomon, G. Grynberg, Europhys. Lett. 21, 13 (1993).

[8] B. Lounis, P. Verkerk, J.-Y. Courtois, C. Salomon, G. Grynberg, Nonlinear Opt. 5, 459 (1993).

[9] A. Hemmerich, M. Weidenmuller, T. Hansch, Europhys. Lett. 27, 427 (1994).

[10] J.W.R. Tabosa, S.S. Vianna, F.A.M. de Oliveira, Phys. Rev. A 55, 2968 (1997). 
[11] G.C. Cardoso, V.R. de Carvalho, S.S. Vianna, J.W.R. Tabosa, Phys. Rev. A 59, 1408 (1999).

[12] G.C. Cardoso, J.W.R. Tabosa, Opt. Commun. 185, 353 (2000).

[13] A. Lezama, G.C. Cardoso, J.W.R. Tabosa, Phys. Rev. A 63, 013805 (2000).

[14] S.M. Wandzura, Opt. Lett. 4, 208 (1979); L.M. Humphrey, J.P. Gordon, P.F. Liao, Opt. Lett. 5, 56 (1980); M. Ducloy, D. Bloch, J. Phys. (Paris) 42, 711 (1981).

[15] M. Mitsunaga, M. Yamashita, M. Koashi, N. Imoto, Opt. Lett. 23, 840 (1998).

[16] J. Zachorowski, T. Pałasz, W. Gawlik, Opt. Appl. 28, 239 (1998).

[17] F.Y. Wu, S. Ezekiel, M. Ducloy, B.R. Mollow, Phys. Rev. Lett. 38, 1077 (1977).

[18] D.J. Harter, R.W. Boyd, IEEE J. Quantum Electron. QE-16, 1126 (1980).

[19] L. Guidoni, P. Verkerk, J. Opt. B, Quantum Semiclass. Opt. 1, R23 (1999).

[20] P. Verkerk, Phys. Rev. Lett. 72, 3017 (1994). 